\title{
Efeitos da Redução da Gordura Epicárdica na Duração da Onda P de Obesos Mórbidos Submetidos à Cirurgia Bariátrica: um Estudo Observacional
}

\author{
Effects of Epicardial Fat Reduction on P-wave Duration of Morbidly Obese \\ Patients Submitted to Bariatric Surgery: an Observational Study \\ Acácio Fernandes-Cardoso $0^{1, *}$, Meive Santos-Furtado², José Grindler ${ }^{1}$, Alfredo José Fonseca'1,
Carlos Rodrigues Oliveira ${ }^{1}$, Nemer Luiz Pichara' ${ }^{1}$, Roberto Cleva ${ }^{3}$, Marco Aurélio Santo ${ }^{3}$ \\ ORCID IDS
}

$\begin{array}{ll}\text { Cardoso AF (D) https://orcid.org/0000-0002-4613-4181 } & \text { Oliveira CR (D) https://orcid.org/0000-0003-1971-5156 } \\ \text { Furtado MS (D) https://orcid.org/0000-0002-9051-0877 } & \text { Pichara NL (D) https://orcid.org/0000-0001-7175-3593 } \\ \text { GrindlerJ (D) https://orcid.org/0000-0001-6646-6469 } & \text { Cleva R (D) https://orcid.org/0000-0001-8673-9014 } \\ \text { Fonseca AJ (D) https://orcid.org/0000-0001-8632-5428 } & \text { Santo MA (D) https://orcid.org/0000-0002-7813-6210 }\end{array}$

\begin{abstract}
RESUMO
Introdução: A gordura epicárdica (GE) é biologicamente ativa e, por meio de seu efeito parácrino, interage com o miocárdio atrial e pode estar envolvida no remodelamento atrial observado em obesos. A duração da onda P (DOP) é um marcador não invasivo do tempo de condução atrial e reflete alterações relacionadas ao remodelamento atrial. Os efeitos da redução da GE induzida pela cirurgia bariátrica sobre a DOP ainda não foram definidos. Métodos: Recrutamos prospectivamente 22 obesos mórbidos sem outras comorbidades na Unidade de Cirurgia Bariátrica do Hospital das Clínicas da Universidade de São Paulo. Os pacientes foram submetidos a avaliações clínica e laboratorial, além de eletrocardiograma (ECG) de 12 derivações, ecocardiograma bidimensional e Holter de $24 \mathrm{~h}$. A mesma avaliação foi realizada 12 meses após a cirurgia bariátrica. A fim de que as variáveis contínuas fossem comparadas, foram utilizados os testes T pareado e de Wilcoxon. Já para avaliar a associação entre variáveis independentes foi utilizado um modelo de regressão para medidas repetidas. Resultados: Ao todo, 20 pacientes completaram o protocolo (idade: $36,35 \pm 10,26$ anos, 18 mulheres). Houve uma redução significativa da DOP, do índice de massa corporal (IMC) e da GE após cirurgia bariátrica $(p<0,05)$. Houve também redução média de 11,55 \pm 8,49 ms na DOP. Na análise de regressão múltipla, foi observada associação entre a redução da DOP e a redução da GE e do IMC. Conclusões: Em obesos mórbidos sem outras comorbidades, a redução da GE após cirurgia bariátrica foi associada a melhora do remodelamento atrial, indicada por uma redução significativa da DOP.
\end{abstract}

PALAVRAS-CHAVE: Onda P; Obesidade Mórbida; Cirurgia Bariátrica.

\begin{abstract}
Introduction: Epicardial fat (EF) is biologically active and, through its paracrine effect, interacts with the atrial myocardium and may be involved in the atrial remodeling observed in obese individuals. P-wave duration (PWD) is a non-invasive marker of atrial conduction time and reflects changes related to atrial remodeling. The effects of the reduction of EF induced by bariatric surgery on PWD have not yet been defined. Methods: We prospectively recruited 22 morbidly obese patients with no other comorbidities at the Unidade de Cirurgia Bariátrica (Bariatric Surgery Unit) of Unviversidade de São Paulo's Hospital das Clínicas. The patients were submitted to clinical and laboratorial evaluations, 12-lead eletrocardiography (ECG), two-dimensional echocardiogram and $24 \mathrm{~h}$ Holter. The same evaluation was performed 12 months after bariatric surgery. In order to make a comparison of the continuous variables, we used the paired and Wilcoxon T tests. To evaluate the association between independent variables, a regression model was used for repeated measures. Results: $A$ total of 20 patients completed the protocol (age: $36.35 \pm 10.26$ years, 18 women). There was a significant reduction of PWD, body mass index $(\mathrm{BMI})$ and $\mathrm{EF}$ after bariatric surgery $(\mathrm{p}<0.05)$. There was also an average reduction of $11.55 \pm 8.49$ ms in PWD. In the multiple regression analysis, an association was observed between the reduction of PWD and the reduction of EF and BMI. Conclusions: In morbidly obese patients with no other comorbidities, the reduction of EF after bariatric surgery was associated with an improvement in atrial remodeling indicated by a significant reduction in PWD.
\end{abstract}

KEYWORDS: P Wave; Morbid Obesity; Bariatric Surgery.

1.Universidade de São Paulo - Faculdade de Medicina - Departamento de Clínica Médica - São Paulo/SP - Brasil.

2. Universidade de São Paulo - Faculdade de Medicina - Instituto de Radiologia -São Paulo/SP - Brasil.

3. Universidade de São Paulo - Faculdade de Medicina - Departamento de Gastroenterologia - São Paulo/SP - Brasil.

*Autor correspondente: acacio.cardoso@hc.fm.usp.br

Recebido: 18 Jul 2019 | Aceito: 14 Ago 2019

Editor Associado: José Tarcísio Medeiros de Vasconcelos 


\section{INTRODUÇÃO}

A gordura epicárdica (GE) é uma camada de tecido adiposo localizada entre a superfície do miocárdio e o pericárdio visceral. Ela cobre $80 \%$ da superfície cardíaca e é responsável por $20 \%$ do peso total do coração, sendo encontrada principalmente nos sulcos atrioventricular e interventricular, em torno dos átrios e ao longo das coronárias ${ }^{1,2}$. Em condições fisiológicas normais, exerce um papel cardioprotetor, por meio da liberação de energia para o miocárdio em situações de alta demanda metabólica. Além disso, participa da termorregulação, atua como anteparo para gânglios e nervos e na regulação da vasomotricidade da circulação coronariana ${ }^{3}$.

Em obesos, a espessura e o volume da GE são reconhecidamente maiores ${ }^{4}$. $\mathrm{O}$ excesso de $\mathrm{GE}$ nessa população tem sido associado à fisiopatologia de algumas doenças cardiovasculares, como a síndrome metabólica, a doença arterial coronariana e a fibrilação atrial ${ }^{5-7}$. Por ser biologicamente ativa e apresentar ampla interação com o miocárdio atrial, especula-se que a GE poderia produzir uma série de mediadores pro-inflamatórios capazes de induzir o desencadeamento de fibrose atrial em obesos ${ }^{8}$. A duração da onda $\mathrm{P}$ do eletrocardiograma (ECG) é reconhecidamente um marcador de remodelamento atrial e está associada ao maior risco de fibrilação atrial ${ }^{9}$. Recentemente, nosso grupo demonstrou que, em obesos mórbidos, a espessura da GE foi independentemente associada a maior duração da onda $\mathrm{P}$, reforçando o impacto negativo da GE no remodelamento atrial ${ }^{10}$. O objetivo do presente estudo foi avaliar os efeitos da redução da GE na duração da onda $\mathrm{P}$ após perda de peso induzida por cirurgia bariátrica.

\section{MÉTODOS População do Estudo}

Entre março de 2015 e fevereiro de 2016, recrutamos consecutivamente 22 obesos mórbidos na Unidade de Cirurgia Bariátrica do Hospital das Clínicas da Faculdade de Medicina da Universidade de São Paulo (HCFMUSP). Na semana que antecedeu a cirurgia bariátrica, os participantes foram submetidos a avaliação antropométrica, história clínica, exame físico, testes laboratoriais, além de ECG de 12 derivações, Holter de 24 h e ecocardiograma bidimensional. A mesma avaliação, com exceção do Holter de 24 h, foi repetida 12 meses após a cirurgia bariátrica. Pacientes com hipertensão arterial, diabetes, dislipidemia, presença de cardiopatias, bloqueios de ramo, história de fibrilação ou flutter atrial, presença de arritmias significativas no Holter de $24 \mathrm{~h}$, anemia ou disfunção tireoidiana e que não completaram o protocolo de avaliação foram excluídos da amostra (Fig. 1). O comitê de ética aprovou o protocolo do estudo e todos os participantes assinaram o termo de consentimento livre e esclarecido.

22 obesos mórbidos (IMC: $46,97 \pm 6,45 \mathrm{~kg} / \mathrm{m}^{2}$, 18 mulheres) foram recrutados entre $03 / 2015$ e $02 / 2016$

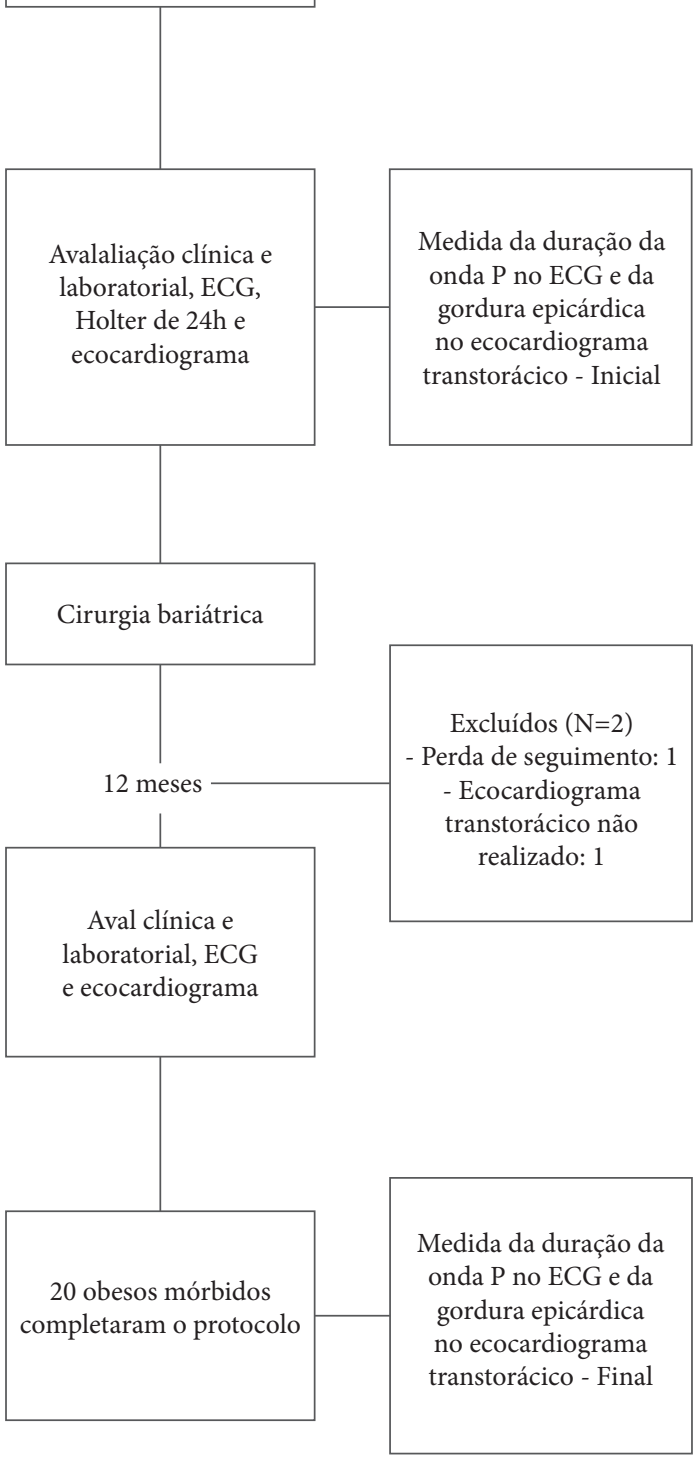

Figura 1. Fluxograma do estudo. 


\section{Análise do Ecocardiograma}

Um ecocardiograma bidimensional (Artida ${ }^{\circledR}$, Toshiba Medical Systems) foi realizado em repouso pelo mesmo observador, com a utilização de um transdutor PST-25BT 1.8-4.2 MHz, no pré-operatório e 12 meses após a cirurgia bariátrica. Com os pacientes em decúbito lateral esquerdo, através dos eixos longo e curto paraesternais longitudinais, visão apical quatro e duas câmaras, os seguintes parâmetros foram acessados: o diâmetro atrial esquerdo, os diâmetros sistólico e diastólico do ventrículo esquerdo (VE) e da cavidade ventricular direita, a espessura do septo interventricular e da parede posterior do VE. A fração de ejeção do VE foi estimada pelo método biplanar de Teichholz. Para avaliar a função diastólica, foram levadas em consideração as velocidades do fluxo mitral por Doppler pulsátil, a fim de determinar as ondas $\mathrm{E}$ e $\mathrm{A}$. A relação $\mathrm{E} / \mathrm{A}$ foi utilizada para avaliar a função diastólica. A massa do VE foi calculada pela fórmula de Devereux.

\section{Avaliação da Gordura Epicárdica}

A espessura da GE foi definida conforme método previamente descrito e validado ${ }^{11}$. Na parede livre do ventrículo direito (VD), durante a fase final da sístole, utilizando o septo interventricular e o anel aórtico como referências anatômicas, a GE foi obtida durante três ciclos cardíacos consecutivos nas incidências paraesternais longitudinal e transversal. O espaço entre a superfície miocárdica e o pericárdio visceral foi definida como sendo GE. A média das medidas registradas nas duas incidências foi considerada o resultado final da espessura da GE. Para avaliar a reprodutibilidade do método, as imagens foram transferidas para uma estação de trabalho, onde um segundo observador, cego em relação à condição clínica dos pacientes, reavaliou a medida da GE. Considerando o total de medidas realizadas no pré- e no pós-operatório, foram sorteados $50 \%$ da amostra para realização da análise interobservador. Uma boa correlação entre os observadores foi demonstrada $(\mathrm{g}=0,9$; IC $95 \%$ $[0,8-1,0])$.

\section{Avaliação da Duração da Onda P no ECG}

Um ECG com registro simultâneo das 12 derivações foi realizado no padrão $2 N$ (velocidade do registro de $50 \mathrm{~mm} / \mathrm{s}$ e amplitude de $2 \mathrm{mV} / \mathrm{cm}$ ) em todos os participantes. Todos os ECG foram obtidos no mesmo aparelho e no mesmo período (entre $10 \mathrm{~h}$ e $12 \mathrm{~h}$ da manhã), a fim de que fossem evitadas variações circadianas dos intervalos eletrocardiográficos. O exame foi repetido, nas mesmas condições, 12 meses após a cirurgia bariátrica. A análise da duração da onda $\mathrm{P}$ foi realizada por um único observador, com experiência no método e cego em relação à condição clínica dos participantes. Para que o registro pudesse ser levado em consideração na análise, eram necessárias no mínimo nove derivações em que a onda $\mathrm{P}$ fosse visível e passível de mensuração. A medida foi realizada manualmente com auxílio de um paquímetro digital com precisão $0,01 \mathrm{~mm}$ e lentes de aumento, como previamente descrito por outros investigadores ${ }^{12}$. O início e o fim da onda $\mathrm{P}$ foram definidos pela junção entre a linha isoelétrica do ECG e as fases inicial e final da onda P. A média da soma de três medidas consecutivas na mesma derivação foi calculada em todas as derivações do ECG (Fig. 2). A maior duração da onda $\mathrm{P}$ no ECG foi utilizada para análise. Uma duração da onda $\mathrm{P}$ maior ou igual a $110 \mathrm{~ms}$ foi considerada aumentada.

\section{Análise Estatística}

Variáveis contínuas foram expressas como média e desvio-padrão, enquanto variáveis categóricas foram apresentadas como números ou proporções. De modo a testar a normalidade, foi utilizado o teste de Kolmogorov-Smirnov.

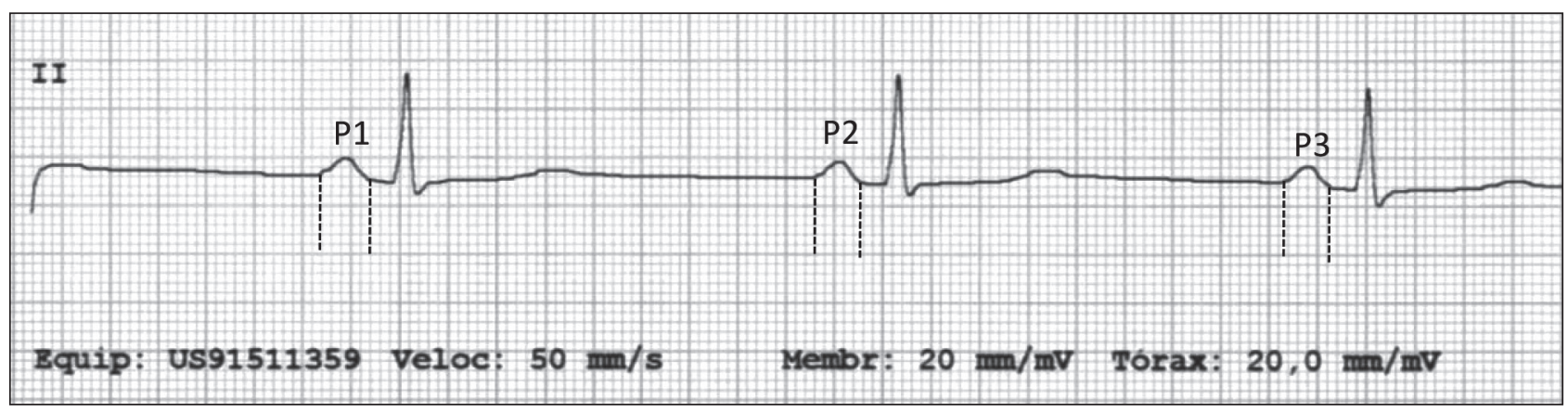

Figura 2. Análise da duração da onda P no ECG (derivação DII). 
Já para comparar variáveis contínuas, foram utilizados os testes $\mathrm{T}$ pareado e de Wilcoxon, conforme a distribuição da população quanto à curva de normalidade. A fim de que fosse avaliada a associação entre variáveis independentes, foi utilizado modelo de regressão para medidas repetidas (equação de estimativas generalizadas; do inglês, Generalized Estimating Equation). Por último, com o objetivo de testar a variabilidade interobservador, foi utilizado o método gama. Um valor de $\mathrm{p}$ abaixo de 0,05 foi considerado estatisticamente significativo. Os dados foram analisados no software $\mathrm{R}$ versão 3.4.1 (R Core Team; Viena, Áustria, 2014).

\section{RESULTADOS}

No total, 20 pacientes completaram o protocolo do estudo (idade: $36,35 \pm 10,26$ anos, 18 mulheres), 17 realizaram a técnica de by-pass gástrico e 3 foram submetidos a gastrectomia vertical por videolaparoscopia (ver Fig. 1). Nenhuma complicação pós-operatória foi observada entre os participantes. Ao longo do seguimento, não foi observada nenhuma intercorrência clínica relacionada à cirurgia.

Os dados clínicos e laboratoriais são demonstrados na Tabela 1. Na avaliação pós-operatória, foi observada redução significativa do peso e do índice de massa corporal (IMC) $(\mathrm{p}<0,001)$. Houve melhora significativa do perfil glicêmico e lipídico, bem como dos níveis de proteína $\mathrm{C}$ reativa $(\mathrm{PCR})$ $(\mathrm{p}<0,05)$. Os níveis de creatinina mantiveram-se inalterados.

As medidas da GE e da duração da onda $P$ são mostradas na Tabela 2. Houve redução significativa da espessura da GE e da duração da onda $\mathrm{P}$ após a cirurgia bariátrica $(7,72 \pm 1,60$ $\times 4,56 \pm 1,40$ e 109,55 $\pm 11,52 \times 98,00 \pm 1,49 ; \mathrm{p}<0,001)$, além de redução média de $11,55 \pm 8,49$ ms na duração da onda $\mathrm{P}$, sendo observada redução acima de $10 \mathrm{~ms}$ em 11 participantes do estudo (55\% da amostra). Antes da cirurgia bariátrica, 8 indivíduos (40\% da amostra) apresentavam duração aumentada da onda P no ECG ( $\geq 110 \mathrm{~ms}$ ) e apenas 3 permaneceram com valores alterados após a cirurgia bariátrica (Fig. 3). Na análise de regressão múltipla, foi observada associação entre as reduções da espessura da GE e do IMC e a redução da duração da onda P no ECG (Tabela 3).

Tabela 1. Características clínicas e laboratoriais da população do estudo.

\begin{tabular}{lccc}
\hline \multicolumn{1}{c}{ Dados } & Antes da cirurgia bariátrica & 12 $\mathrm{m}$ após a cirurgia bariátrica & Valor de $\mathrm{p}$ \\
\hline Peso $(\mathrm{kg})$ & $126,95 \pm 16,38$ & $89,47 \pm 17,55$ & $<0,001$ \\
Índice de massa corporal $\left(\mathrm{kg} / \mathrm{m}^{2}\right)$ & $47,19 \pm 6,15$ & $33,08 \pm 6,91$ & $<0,001$ \\
Pressão arterial sistólica $(\mathrm{mmHg})$ & $110,10 \pm 12,71$ & $108,50 \pm 7,96$ & 0,224 \\
Pressão arterial diastólica $(\mathrm{mmHg})$ & $74,45 \pm 6,80$ & $74,45 \pm 5,25$ & 0,344 \\
Glicemia (mg/dl) & $88,10 \pm 9,30$ & $82,6 \pm 4,36$ & 0,019 \\
Hemoglobina glicada (\%) & $5,45 \pm 0,43$ & $5,18 \pm 0,40$ & 0,003 \\
Colesterol total (mg/dl) & $184,85 \pm 23,30$ & $151,60 \pm 17,97$ & 0,001 \\
Triglicérides (mg/dl) & $121,10 \pm 50,24$ & $76,80 \pm 21,33$ & 0,001 \\
Proteína C reativa (mg/dl) & $2,63 \pm 2,14$ & 0,001 \\
Creatinina (mg/dl) & $11,79 \pm 7,64$ & $0,74 \pm 0,14$ & 0,622 \\
\hline
\end{tabular}

Tabela 2. Dados das medidas da duração da onda P e do ecocardiograma.

\begin{tabular}{|c|c|c|c|}
\hline Dados & Antes da cirurgia bariátrica & $12 \mathrm{~m}$ após a cirurgia bariátrica & Valor de $p$ \\
\hline Duração da onda P (ms) & $109,55 \pm 11,52$ & $98,00 \pm 10,49$ & 0,001 \\
\hline Diâmetro do átrio esquerdo (mm) & $36,12 \pm 3,46$ & $37,06 \pm 2,73$ & 0,40 \\
\hline Diâmetro sistólico do ventrículo esquerdo (mm) & $30,30 \pm 2,49$ & $30,52 \pm 1,51$ & 0,742 \\
\hline Diâmetro diastólico do ventrículo esquerdo (mm) & $46,20 \pm 4,23$ & $47,70 \pm 2,11$ & 0,137 \\
\hline Fração de ejeção do ventrículo esquerdo (\%) & $63,15 \pm 4,25$ & $65,65 \pm 2,85$ & 0,016 \\
\hline Parede posterior (mm) & $9,40 \pm 0,99$ & $9,26 \pm 0,94$ & 0,524 \\
\hline Septo (mm) & $9,45 \pm 1,0$ & $9,61 \pm 0,99$ & 0,606 \\
\hline $\mathrm{E} / \mathrm{A}(\mathrm{cm} / \mathrm{s})$ & $1,31 \pm 0,39$ & $1,60 \pm 0,48$ & $<0,001$ \\
\hline Massa do ventrículo esquerdo ( $\left.\mathrm{g} / \mathrm{m}^{2}\right)$ & $151,10 \pm 27,73$ & $158,05 \pm 22,35$ & 0,391 \\
\hline Gordura epicárdica (mm) & $7,72 \pm 1,60$ & $4,56 \pm 1,40$ & $<0,001$ \\
\hline
\end{tabular}




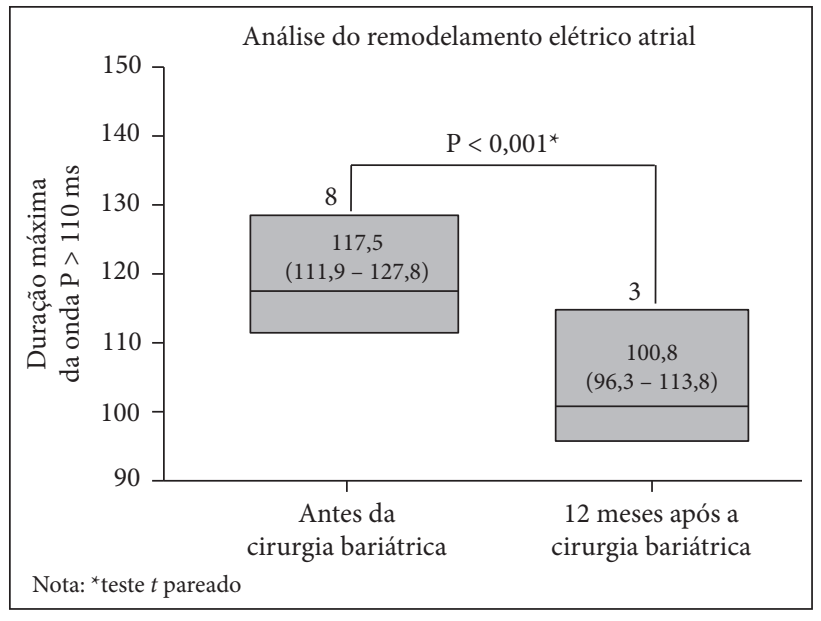

Figura 3. Avaliação do remodelamento elétrico atrial para obesos com duração da onda P acima de 110 ms.

Tabela 3. Análise de regressão múltipla.

\begin{tabular}{ccccc}
\hline & $\boldsymbol{\beta}$ & Dp & $\begin{array}{c}\text { Valor } \\
\text { de } \mathbf{p}\end{array}$ \\
\hline $\begin{array}{c}\text { Duração } \\
\text { da onda P }\end{array}$ & $\begin{array}{c}\text { Gordura } \\
\text { epicárdica } \\
\text { Índice de } \\
\text { massa corporal }\end{array}$ & 0,96 & 1,36 & 0,029 \\
& 0,47 & 0,22 & 0,038 \\
\hline
\end{tabular}

\section{DISCUSSÃO}

Neste estudo, observamos que a redução da GE promovida pela perda de peso após cirurgia bariátrica foi associada à diminuição significativa da duração da onda $\mathrm{P}$. Esse achado envolve a gordura visceral cardíaca no processo fisiopatológico de remodelamento atrial nos obesos mórbidos.

O impacto da obesidade e da GE no remodelamento elétrico e estrutural dos átrios já foi testado em estudos experimentais. Em um modelo animal, Abed et al. demonstraram que o aumento progressivo da obesidade foi capaz de promover aumento do volume dos átrios, da inflamação e do grau de fibrose intersticial atrial, bem como do acúmulo de gordura no miocárdio e da expressão de receptores atriais pró-fibróticos ${ }^{13}$. Já no estudo conduzido por Lin et al., a incubação da célula atrial por adipócitos do epicárdio foi capaz de prolongar o potencial de ação, alterar a funcionalidade dos canais iônicos e aumentar a indução de atividade deflagrada quando essas células foram expostas ao efeito do isoproterenol, demonstrando que a gordura visceral pode modular as propriedades eletrofisiológicas da célula atrial e contribuir para o remodelamento elétrico dos átrios ${ }^{14}$. Mais recentemente, Mahajan et al., observaram que mudanças eletrofisiológicas na parede posterior de ovelhas obesas foram atribuídas exclusivamente à infiltração da GE, indicando que esse fenômeno localizado pode contribuir para a formação de substrato arritmogênico nos átrios ${ }^{15}$.

A associação entre GE e remodelamento atrial também é corroborada por evidências em registros populacionais e estudos observacionais. Segundo no Framingham Heart Study, a gordura pericárdica foi associada a medidas eletrocardiográficas relacionadas à condução atrial (duração da onda $\mathrm{P}$, intervalo $\mathrm{PR}$ e força terminal da onda $\mathrm{P}$ ) mesmo após ajustes para depósitos gordurosos extracardíacos ${ }^{16}$. Em população semelhante à do nosso estudo, Iacobellis et al. demonstraram uma correlação entre as dimensões do átrio esquerdo (AE) medidas por ecocardiograma e a espessura da $\mathrm{GE}^{17}$. Em análises envolvendo ECG e diferentes métodos de imagem, a duração da onda $\mathrm{P}$ foi associada à espessura e ao volume da $\mathrm{GE}^{10-18}$. Em conjunto, todas essas análises reforçam a hipótese do envolvimento da gordura visceral cardíaca no remodelamento dos átrios.

O impacto da redução do peso no remodelamento cardíaco é conhecido e já foi analisado após cirurgia bariátrica ${ }^{19}$. Estudos observacionais também indicam que a redução do peso é capaz de promover redução da duração e da dispersão da onda $\mathrm{P}^{20}$. No entanto, as evidências que indicam os efeitos da redução da GE no remodelamento dos átrios ainda são escassas. No estudo conduzido por Monno et al., o remodelamento reverso dos átrios foi avaliado em um grupo de pacientes submetidos a ablação por cateter e foi inversamente associado à maior espessura da GE e à presença de síndrome metabólica ${ }^{21}$. Embora não tenha havido redução do peso ao longo do seguimento, foi observada redução significativa da GE no grupo que não apresentou recorrência de fibrilação atrial (FA) após a ablação. Em nosso estudo, a redução da onda $\mathrm{P}$ foi associada à redução do IMC e da GE. Como são condições interdependentes, a contribuição dessas variáveis em modelos que envolvam a redução do peso e da obesidade deve ser considerada em conjunto, pois tais variáveis dificilmente estarão dissociadas.

Embora a medida da duração da onda $\mathrm{P}$ reflita indiretamente o tamanho dos átrios e a normalização dessa duração tenha ocorrido em $40 \%$ dos obesos, neste estudo não evidenciamos redução do diâmetro do $\mathrm{AE}$ após cirurgia bariátrica. Questões técnicas relacionadas à aquisição da imagem em pacientes com janela ecográfica limitada podem ter interferido nos resultados e não podem ser desconsideradas. Por outro lado, é possível que as alterações observadas reflitam mais uma melhora da condução do que 
necessariamente uma redução do diâmetro atrial. Avaliações do remodelamento atrial por mapeamento eletroanatômico demonstraram que, em áreas de baixa voltagem, a presença de potenciais fragmentados e de alentecimento da condução atrial foram associadas a regiões de depósito de GE nos átrios de pacientes obesos ${ }^{22}$. Quão modificáveis, por mudanças no estilo de vida e intervenções como a cirurgia bariátrica, são essas alterações, é motivo para futuras investigações.

As limitações do presente estudo estão relacionadas principalmente, ao tamanho e ao caráter restritivo da amostra. A inclusão apenas de obesos sem outras comorbidades, embora diminua a validação externa do estudo, foi importante para atenuar possíveis vieses de confusão presentes em análises que envolvem avaliação do remodelamento atrial e obesidade. Atualmente, a mensuração do volume da GE é mais bem-estimada pela tomografia ou pela ressonância magnética, porém a disponibilidade desses métodos diagnósticos para obesos mórbidos é limitada. A mensuração da onda $P$ por ECG realizada manualmente pode apresentar acurácia limitada quando comparada a outros métodos de avaliação ${ }^{23}$. Novos estudos são necessários para confirmar nossos achados.

\section{CONCLUSÕES}

Em um seleto grupo de obesos mórbidos submetidos à cirurgia bariátrica a redução da espessura da GE foi associada à redução da duração da onda $\mathrm{Pe}$ indica que a gordura visceral cardíaca e a redução do peso podem ter um papel relevante no remodelamento elétrico reverso dos átrios.

\section{CONTRIBUIÇÃO DOS AUTORES}

Conceituação, Cardoso AF, Grindler J, Santo MA; Metodologia, Cardoso AF, Furtado MS, Fonseca AJ, Pichara NL, Oliveira CR, Cleva R; Investigação, Cardoso AF, Furtado MS, Santo MA; Redação primeira versão, Cardoso AF, Cleva R, Santo MA; Redação - Revisão \& Edição, Cardoso AF, Santo MA; Supervisão, Cardoso AF, Santo MA.

\section{REFERÊNCIAS}

1. Iacobellis G, Corradi D, Sharma AM. Epicardial adipose tissue: anatomic, biomolecular and clinical relationships with the heart. Nat Clin Pract Cardiovasc Med 2005 Oct;2(10):536-43. https://doi.org/10.1038/ncpcardio0319

2. Corradi D, Maestri R, Callegari S, Pastori P, Goldoni M, Luong TV, et al. The ventricular epicardial fat is related to the myocardial mass in normal, ischemic and hypertrophic hearts. Cardiovasc Pathol 2004 Nov;13(6):313-6. https://doi. org/10.1016/j.carpath.2004.08.005

3. Sacks HS, Fain JN. Human epicardial fat: what is new and what is missing? Clin Exp Pharmacol Physiol 2011 Dec 1;38(12):879-87. https://doi.org/10.1111/j.14401681.2011.05601.x

4. Cikim AS, Topal E, Harputluoglu M, Keskin L, Zengin Z, Cikim K, et al. Epicardial adipose tissue, hepatic steatosis and obesity. J Endocrinol Invest. Springer International Publishing 2007 Jun;30(6):459-64. https://doi.org/10.1007/ BF03346328

5. Lyon CJ, Law RE, Hsueh WA. Minireview: adiposity, inflammation, and atherogenesis. Endocrinology 2003 Jun;144(6):2195-200. https://doi.org/10.1210/en.2003-0285

6. Eckel RH, Grundy SM, Zimmet PZ. The metabolic syndrome. Lancet 2005 Apr;365(9468):1415-28. htpps://doi. org/10.1016/S0140-6736(05)66378-7
7. Bos D, Vernooij MW, Shahzad R, Kavousi M, Hofman A, van Walsum $T$, et al. Epicardial fat volume and the risk of atrial fibrillation in the general population free of cardiovascular disease. JACC Cardiovasc Imaging 2017 Nov;10(11):1405-7. https://doi.org/10.1016/j.jcmg.2016.12.005

8. Auer J. Fat: an emerging player in the field of atrial fibrillation. European heart journal 2017;38:62-5. https:// doi.org/10.1093/eurheartj/ehw013

9. Hari KJ, Nguyen TP, Soliman EZ. Relationship between P-wave duration and risk of atrial fibrillation. Expert Rev Cardiovasc Ther2018 Nov;16(11):837-43. https://doi.org/10 $.1080 / 14779072.2018 .1533814$

10. Fernandes-Cardoso A, Santos-Furtado M, Grindler J, Ferreira LA, Andrade JL, Santo MA. Epicardial fat thickness correlates with P-wave duration, left atrial size and decreased left ventricular systolic function in morbid obesity. Nutr Metab Cardiovasc Dis 2017 Aug;27(8):731-8. https://doi. org/10.1016/j.numecd.2017.05.009

11. Iacobellis G, Willens HJ. Echocardiographic epicardial fat: a review of research and clinical applications. J Am Soc Echocardiogr 2009 Dec;22(12):1311-9;1417-8. https://doi. org/10.1016/j.echo.2009.10.013

12. Kosar F, Aksoy Y, Ari F, Keskin L, Sahin I. P Wave duration and dispersion in obese subjects. Ann Noninvas Electro 2008 Jan 1;13(1):3-7. https://doi.org/10.1111/j.1542474X.2007.00194.X 
13. Abed HS, Samuel CS, Lau DH, Kelly DJ, Royce SG, Alasady $M$, et al. Obesity results in progressive atrial structural and electrical remodeling: implications for atrial fibrillation. Heart Rhythm 2013 Jan;10(1):90-100. https://doi.org/10.1016/j. hrthm.2012.08.043

14. Lin YK, Chen YC, Chen JH, Chen SA, Chen YJ. Adipocytes modulate the electrophysiology of atrial myocytes: implications in obesity-induced atrial fibrillation. Basic Res Cardiol2012 Sep;107(5):293. https://doi.org/10.1007/s00395-012-0293-1

15. Mahajan R, Lau DH, Brooks AG, Shipp NJ, Manavis J, Wood JPM, et al. Electrophysiological, electroanatomical, and structural remodeling of the atria as consequences of sustained obesity. J Am Coll Cardiol 2015 Jul 7;66(1):1-11. https://doi.org/10.1016/j. jacc.2015.04.058

16. Friedman DJ, Wang N, Meigs JB, Hoffmann U, Massaro JM, Fox CS, et al. Pericardial fat is associated with atrial conduction: the Framingham Heart Study. J. Am. Heart Assoc 2014 Apr 22;3(2):e000477-7. https://doi.org/10.1161/JAHA.113.000477

17. lacobellis G, Leonetti F, Singh N, Sharma AM. Relationship of epicardial adipose tissue with atrial dimensions and diastolic function in morbidly obese subjects. Int J Cardiol 2007 Feb;115(2):272-3. https://doi.org/10.1016/j.ijcard.2006.04.016

18. Jhuo SJ, Hsieh TJ, Tang WH, Tsai WC, Lee KT, Yen HW, et al. The association of the amount of epicardial fat, P wave duration, and PR interval in electrocardiogram. J Electrocardiol 2018 JulAug;51(4):645-51. https://doi.org/10.1016/j.jelectrocard.2018. 04.009

19. Tuluce K, Kara C, Tuluce SY, Cetin N, Topaloglu C, Bozkaya YT, et al. Early reverse cardiac remodeling effect of laparoscopic sleeve gastrectomy. Obes Surg 2017 Feb;27(2):364-75. https:// doi.org/10.1007/s11695-016-2301-2

20. Falchi AG, Grecchi I, Muggia C, Tinelli C. Weight loss and P wave dispersion: a preliminary study. Obes Res Clin Pract 2014 NovDec;8(6):e614-7. https://doi.org/10.1016/j.orcp.2014.08.005

21. Monno K, Okumura Y, Saito Y, Aizawa Y, Nagashima K, Arai M, et al. Effect of epicardial fat and metabolic syndrome on reverse atrial remodeling after ablation for atrial fibrillation. J Arrhythm 2018 Oct 13;34(6):607-16. https://doi.org/10.1002/joa3.12124

22. Mahajan R, Nelson A, Pathak RK, Middeldorp ME, Wong CX, Twomey DJ, et al. Electroanatomical remodeling of the atria in obesity: impact of adjacent epicardial fat. JACC Clin Electrophisiol 2018 Dec;4(12):1529-40. https://doi. org/10.1016/j.jacep.2018.08.014

23. Dilaveris P, Batchvarov V, Gialafos J, Malik M. Comparison of different methods for manual $P$ wave duration measurement in 12-lead electrocardiograms. Pacing Clin Electrophysiol 1999 Oct;22(10):1532-8. https://doi.org/10.1111/j.1540-8159.1999. tb00358.x 